\title{
Desarrollo rural y transformaciones del paisaje: Estudio de caso, Cuenca de Tulahuencito. Región de Coquimbo, Chile
}

\section{Rural development and landscape transformations: Case study, Tulahuencito basin. Region of Coquimbo, Chile}

\author{
Eduardo Jaime Muñoz ${ }^{1}$
}

\section{RESUMEN}

Se presenta un estudio geográfico, relacionado con el desarrollo rural y transformación del paisaje en la Cuenca de Tulahuencito, ubicada en la comuna de Monte Patria, Región de Coquimbo, Chile.

El objetivo general de la investigación es conocer y analizar las actividades agrarias y ganaderas que han modificado el entorno natural de la unidad de estudio. Los materiales utilizados fueron hojas de muestreo, máquina fotográfica, grabadora y bibliografía especializada. Los métodos empleados fueron trabajo en terreno, análisis de datos cartográficos y entrevistas. En los resultados se observa una degradación del espacio natural de Tulahuencito, producto de las actividades económicas, como la siembra del grano, el pastoreo de ganado vacuno, caballar y caprino, actividades que se iniciaron junto al desarrollo del modelo exportador de materias primas (1830-1930), continuando con el modelo de sustitución de importaciones (1930-1975) y finalmente el modelo de libre mercado (1975 hasta hoy). A modo de conclusión existe degradación en la zona producto de la agricultura y ganadería, que ha dejado en el territorio claros suelos desnudos, afectando la conservación de la biodiversidad.

Palabras Claves: Cuenca; modelos económicos; actividades agrarias; ganadería caprina y degradación.

1 Universidad de Valparaiso, Chile. Universidad Nacional de San Juan. Argentina. Chile jaime.557@hotmail.com ORCID iD: http://orcid.org/0000-0003-4412-0664

Copyright: (0) 2018 CSIC. Este es un artículo de acceso abierto distribuido bajo los términos de la licencia de uso y distribución Creative Commons Reconocimiento 4.0 Internacional (CC BY 4.0). 


\begin{abstract}
A geographical study related to rural development and transformation of the landscape of Tulahuencito Basin, located in the town of Monte Patria, Coquimbo Region, Chile is presented.

The overall objective of the research is to know and analyze agricultural and livestock activities that have altered the natural environment unit of study. The materials used were leaves sampling, camera, recorder and literature. The methods used were field work, mapping data analysis and interviews. Results in a degradation of the natural space Tulahuencito product of economic activities, such as planting grain, grazing cattle, horses and goats, activities initiated by the development of raw materials export model is observed (1830-1930), continuing with the import substitution model (1930-1975) and finally the free market model (1975-present). To conclude there is degradation in the product area of agriculture, which has made clear the land bare soil, affecting the conservation of biodiversity.
\end{abstract}

KEY wORDS: Cuenca; economic degradation models; farming; goat farming and degradation.

Cómo citar este artículo/Citation: Jaime Muñoz, Eduardo (2018): "Desarrollo rural y transformaciones del paisaje: Estudio de caso, cuenca de Tulahuencito. Región de Coquimbo, Chile", Estudios Geográficos, LXXIX/284, pp. 39-59

\title{
INTRODUCCIÓN
}

La importancia que tiene estudiar los cambios que ha sufrido el paisaje natural por efecto de la intervención humana para el desarrollo de sus actividades económicas, en localidades apartadas de centros urbanos, como Tulahuencito, es un aporte al conocimiento de la historia económica local y regional y también es una contribución a la toma de decisiones que involucren el desarrollo rural productivo en la zona semi-árida del país.

El objetivo del estudio es establecer un diagnóstico de las actividades económicas que han transformado el paisaje natural de la Cuenca de Tulahuencito, afectada por el uso intensivo de los terrenos para actividades como: la siembra del grano, el pastoreo de ganado vacuno, caprino, caballar. Un segundo punto a considerar desde la perspectiva geográfica, es el estudio de los componentes que determinan el crecimiento de un lugar sin afectar los recursos naturales, que forman parte del paisaje tradicional de una cuenca, para evitar el desequilibrio ecológico que ello origina ${ }^{2}$.

En cuanto a la hipótesis de trabajo se plantea que «Las actividades agrarias y ganaderas en Tulahuencito, fueron deteriorando paulatinamente el paisaje natural, generando pobreza en su población y una mayor desigualdad social en la Región».

\footnotetext{
2 Sánchez, A. (2005): Geografía de Chile
} 
A través del estudio se abordaran antecedentes que dieron vida al desarrollo productivo de la cuenca desde sus orígenes hasta la actualidad, para intentar dar respuesta a la pregunta: ¿cuáles han sido las actividades económicas que han transformado el paisaje natural en la Cuenca de Tulahuencito?

\section{MARCo TEORICO}

En Chile los problemas de centralización y administración de los recursos, son un reflejo de la estructura natural del Estado chileno, lo que analizado con mayor detención, generan una dificultad para promover el desarrollo rural de las zonas más apartadas, de los centros de toma de decisión. Según La Comisión asesora presidencial en descentralización y desarrollo ${ }^{3}$ plantea «la necesidad de impulsar una política de Estado que promueva la descentralización y el desarrollo local-regional, para alcanzar un bienestar económico y social equilibrado y sustentable. Diversas son las ideas, que se pueden emprender para lograr un crecimiento en las regiones, pero en ello influye el retraso y la falta de una mayor coordinación en las autoridades locales, regionales y nacionales. Todo esto conduce a un crecimiento desigual, siendo necesaria una política comprometida que permita mejorar la calidad de vida de las comunidades que se localizan en zonas apartadas como el caso de Tulahuencito.

Según el Informe Ambiental del Plan Regulador Comunal de Monte Patria ${ }^{4}$, se plantea promover la sustentabilidad ambiental, mejorar la calidad de vida de los ciudadanos, y garantizar un medio ambiente libre de contaminación. Esta medida compromete los centros poblados como Monte Patria con 5.910 habitantes y El Palqui 6.669 habitantes, Huatulame con 991 habitantes, Tulahuén 1665 habitantes, Carén 1.250 y Pedregal localidad cercana a la Cuenca de Tulahuencito con 733 habitantes. Cabe señalar que la economía basada en la actividad caprina y la actividad temporera, es la que da vida y sustento a los habitantes del sector. De acuerdo con el plan de acción de la biodiversidad de la Región de Coquimbo ${ }^{5}$ se propone un objetivo fundamental que es la protección

3 Propuesta de Política de Estado y Agenda para la Descentralización y el Desarrollo Territorial de Chile Hacia un país desarrollado y justo (octubre 2014)

${ }^{4}$ Plan Regulador Comunal de Monte Patria (2015).Región de Coquimbo. Informe Ambiental: "promover la sustentabilidad ambiental del proceso de desarrollo, con miras a mejorar la calidad de vida de los ciudadanos, garantizando un medio ambiente libre de contaminación, la protección del entorno, la preservación de la naturaleza y la conservación del patrimonio ambiental".

5 Comisión Nacional del Medio Ambiente (2008): Estrategia Regional y Plan de acción de la Biodiversidad, IV Región 
del patrimonio ambiental y el uso sustentable de los recursos naturales. Esta acción cobra urgencia en la unidad de estudio, donde es necesario una planificación que permita un mejor manejo de las potencialidades productivas y un desarrollo sustentable para las comunidades de la zona.

2. Ubicación geográfica de la Cuenca de Tulahuencito, comuna de Monte PaTRIA, IV ReGión DE COQUimbo

De acuerdo con el Plan de Desarrollo Comunal (2012-2018) ${ }^{6}$, la estrategia de desarrollo para la región de Coquimbo promueve actividades sustentables valorando la base productiva diversa, impulsando la expansión, modernización, competitividad e internacionalización.

En el caso de la Cuenca de Tulahuencito, hay que considerar que es una unidad territorial precordillerana, posee una superficie de 14.138,83 hectáreas, se encuentra ubicada en la Comuna de Monte Patria, Región de Coquimbo en las siguientes coordenadas geográficas: $30^{\circ} 51^{\prime 22}$. 20 Sur y $70^{\circ} 32 ` 35.02$ Oeste.

\subsection{La siembra del grano: primera actividad agraria en los terrenos de la Cuenca de Tulahuencito.}

La historia económica de Chile, se caracteriza por la existencia de tres estrategias de crecimiento que dieron vida al desarrollo de la nación. El primer modelo que se puso en práctica fue el exportador de materias primas, iniciándose en tiempos de la República en (1830) y culminando el año 1930. La segunda estrategia corresponde al inicio del proceso de industrialización, con ello el, foco se centró en impulsar el desarrollo productivo de la industria a través de la creación de empresas nacionales, y cronológicamente corresponde al periodo 1930 a 1945, aunque en la práctica el quiebre fue en 1975, «con la llegada del gobierno militar, y con ello el inicio del modelo de libre mercado, hasta 1989, a partir de la vuelta a la democracia, el país mantiene una estrategia de crecimiento, que se puede definir como Modelo Social de Mercado» ${ }^{7}$.

${ }^{6}$ El Plan de Desarrollo Comunal, PLADECO, es un instrumento de planificación estratégica y gestión municipal, normado por la Ley Orgánica de Municipalidades No 18.695. Su principal objetivo es ser una respuesta a las demandas sociales, proponiendo prioridades, lineamientos, políticas y planes de acción que coordinen eficazmente instituciones, actores y recursos.

7 Sánchez A. (2015). Geografía de Chile. Santiago de Chile. Biblioteca Internacional. 
FIgURA 1

LA CUENCA DE TULAHUENCITO, SU HIDROGRAFIA Y LAS DIFERENCIAS ALTITUDINALES

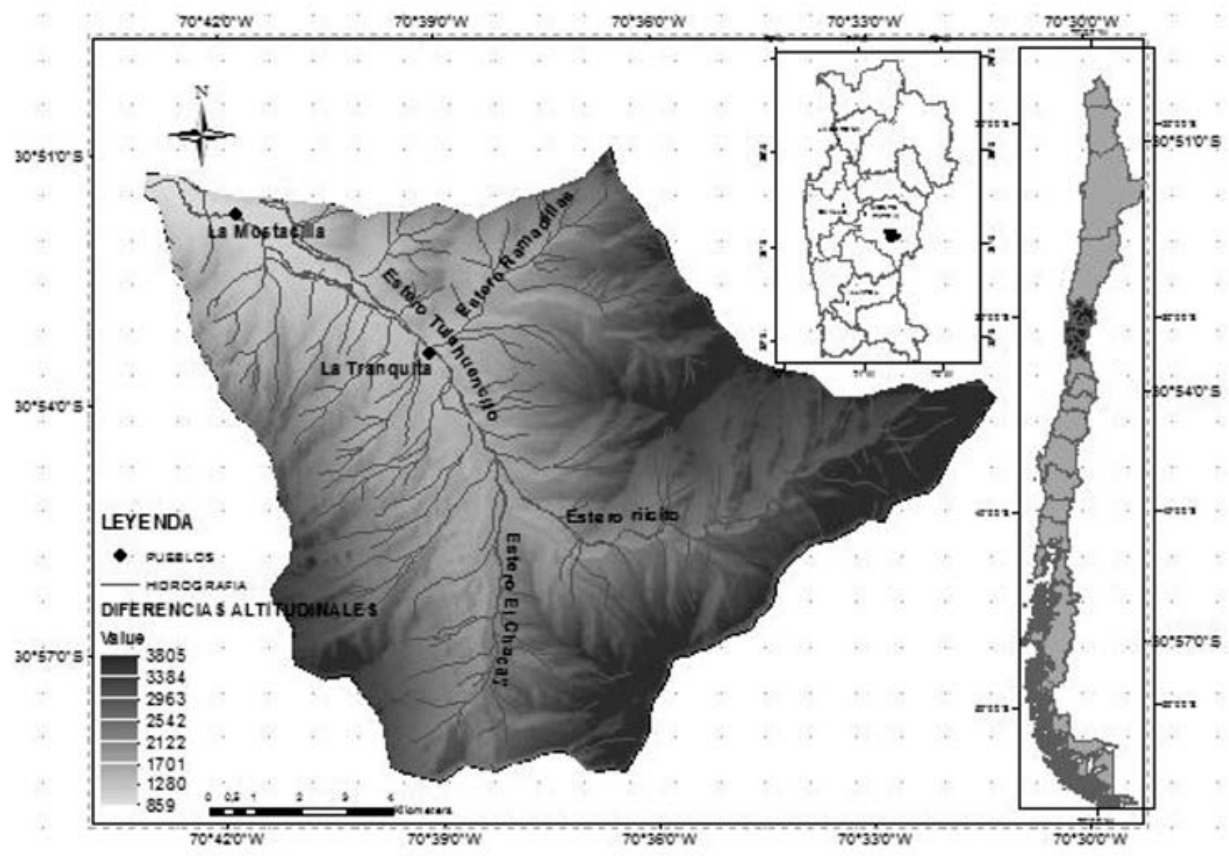

Fuente: CEAZA, (2014).

Los cambios de estrategia económica en la Cuenca de Tulahuencito, fueron transformando el paisaje natural y con ello las condiciones de vida de sus ocupantes. El modelo exportador primario, permitió el desarrollo de una agricultura basada en la siembra del grano, la llegada del modelo de sustitución de importaciones continuó con la producción agrícola y uso del territorio para sembrar el grano y pastar el ganado vacuno y caballar, incorporando la figura del mediero al proceso productivo.

Los cambios de estrategia económica influyeron también en los dueños de las tierras de la cuenca, terminaron por cambiar la forma de producción en el área a manera de ejemplo se puede citar la hacienda La Ortiga, una de las más importantes de la zona, la cual presentaba problemas de administración y una permanente disminución del recursos hídricos, componente muy importante para el desarrollo en la comuna. Esta situación fue cambiando el paisaje de 
Tulahuencito, una progresiva erosión y desertificación del medio ambiente con claros de suelos desnudos, por falta de agua para regar los cultivos y poco interés de los medieros en sembrar, sumado a la caída de los precios del grano, marcó el inicio de una nueva etapa en el área de estudio con la introducción del ganado caprino y el uso de leña para hacer carbón, fueron modificando el paisaje de secano en la zona.

Un segundo aspecto a considerar en la cuenca fueron las variaciones en el número de exportaciones expresadas en toneladas. Las materias primas que Chile vendía al extranjero e importaciones de materias primas para comercializar y mantener la economía local. En este tiempo, las cifras comienzan caer debido al desplome de la economía el año 1929, estos fueron años donde Chile incorpora a su política económica el modelo de sustitución por importaciones, incentivando la creación de industrias en el Chile del siglo XX. A continuación se muestra en el cuadro 1, algunos valores entre los años 1930 a 1940.

\section{TABLA 1}

PRODUCCIÓN NACIONAL DE EXPORTACIONES E IMPORTACIONES DE CHILE EN LOS AÑOS (1930-1940)

\begin{tabular}{c|c|c}
\hline Año & Exportaciones (toneladas) & Importaciones (toneladas) \\
\hline 1930 & 273.593 & 288.425 \\
\hline 1931 & 269.896 & 145.416 \\
\hline 1932 & 59.842 & 44.044 \\
\hline 1933 & 70.817 & 37.454 \\
\hline 1934 & 96.785 & 49.793 \\
\hline 1935 & 97.456 & 62.550 \\
\hline 1936 & 115.827 & 71.427 \\
\hline 1937 & 195.203 & 87.934 \\
\hline 1938 & 140.660 & 102.785 \\
\hline 1939 & 138.299 & 84.613 \\
\hline 1940 & 143.523 & 104.443 \\
\hline
\end{tabular}

Fuente: Toso, R y Feller, A (1983).

En relación a las exportaciones, se observa que enel año 1932 experimentaron una baja, producto de la puesta en marcha del nuevo modelo económico y los procesos políticos ocurridos en el país. «La caída de los precios en los mercados 
externos repercutió dramáticamente en Chile. Entre 1929 y 1932 el valor total de la producción de salitre cayó en un 95\% desde 1.050 millones de pesos en 1929 hasta 58 millones de pesos en $1932 \gg^{8}$. El cambio de las políticas económicas hacia modelos de intervención desencadenó un sentimiento de nacionalismo estatal pero, a pesar de esta situación, el sistema económico chileno continúo su dependencia de los mercados internacionales. Por esta razón, era necesario importar productos, especialmente aquellos que provienen de la actividad cuprífera, todos necesarios para impulsar el desarrollo de la industria nacional.

En esta época de cambios, la economía de la Cuenca de Tulahuencito se basaba en la siembra de productos como el trigo, alfalfa, cebada, maíz, porotos y la crianza de ganado vacuno para comercializar su carne y cuero, mulas y yeguas para ser empleadas en la trilla y el acarreo de los productos de la hacienda hasta sus centros de acopio.

Con los avances de la modernidad del país, estas actividades agrarias fueron permeando la vida de los campesinos en Tulahuencito. Sin embargo la crisis económica de 1929, significó un cambio de modelo económico que desencadenó una nueva forma en la administración de las tierras. Uno de las primeras medidas tomadas por el hacendado fue la creación de la figura del «mediero», denominación que recibe los sembradores que siembran a media con el hacendado.

Una segunda medida fue, priorizar tierras cercanas a los canales, debido la escasez de agua, un caso fue el sector de él «El portezuelo ${ }^{9}$ », en el lugar existe una planicie y laderas cubiertas por espinos y algarrobos, los sembradores fueron despejando y quemando la vegetación para sembrar.

Un tercer aspecto a considerar asociado a la figura del «mediero» en la hacienda La Ortiga, unidad territorial ubicada en la antigua subdelegación de Agua Amarrilla, Departamento de Ovalle, fue la siembra de mayores extensiones de terrenos, cercanos a cursos de agua. «En el sector agrario, en efecto en los momentos de crisis, el latifundista el «hacendado», no despide masivamente su mano de obra manual: la conserva aumentándole, en cambio, las obligaciones de trabajo ${ }^{10}$. Esta estrategia pareció ser implementada en las

8 Aylwin, M., Bascuñán, C., Correa, S., Gazmuri. C., Serrano, S. y Tagle. M. (1994). Chile en el siglo XX. Santiago: Quinta edición Editorial Planeta.

9 Biblioteca del Congreso Nacional de Chile (2017). Glosario de términos geográficos. Significado de "Portezuelo": Sección baja o deprimida situada entre dos relieves mayores. En URL. https://www.bcn.cl/siit/glosario/index_html

10 Carmagnani. M. (1998). Desarrollo industrial y subdesarrollo económico el caso chileno (1860-1920). Santiago, Chile: Dirección de bibliotecas, Archivos y Museos. Departamento de Ciencias Históricas Universidad de Chile y Centro de Investigaciones Diego Barros Aranas. 
haciendas rurales, como la de La Ortiga a través de la figura del «mediero». El hacendado era dueño de la semilla, terreno y el agua, por su parte el mediero sembraba, producía y cosechaba repartiendo a medias los productos con el latifundista, bajo este nuevo sistema se sembró el sector de El Portezuelo, El Gredal, El Espinal, La Quema y La Mostacilla, sectores pertenecientes a la hacienda La Ortiga.

\section{TABLA 2}

TABLA DE CANTIDAD DE TRIGO, ALVERJONES Y POROTOS EXPRESADAS EN FANEGAS PRODUCCIÓN EXISTENTE EN LA CUENCA DE TULAHUENCIO SECTOR EL PORTEZUELO EN LOS AÑOS 1933, 1960 Y 1970

\begin{tabular}{c|c|c|c|c}
\hline Años & $\begin{array}{c}\text { Cantidad } \\
\text { de medieros }\end{array}$ & $\begin{array}{c}\text { Producción } \\
\text { de Trigo }\end{array}$ & $\begin{array}{c}\text { Producción } \\
\text { de Alverjones }\end{array}$ & $\begin{array}{c}\text { Producción } \\
\text { de Porotos }\end{array}$ \\
\hline 1933 & 10 medieros & 295 fanegas & 250 fanegas & 335 fanegas \\
\hline 1960 & 11 medieros & 475 fanegas & 435 fanegas & 365 fanegas \\
\hline 1970 & 7 medieros & 205 fanegas & 205 fanegas & 260 fanegas \\
\hline
\end{tabular}

Fuente: Jaime P. (2014).

Nota: Fanega: es una unidad de medida, donde 1 fanega: 12 decalitros.

El uso de territorio para la siembra favoreció el aprovechamiento de suelos fértiles, las cosechas obtenidas por los medieros en el sector «El Portezuelo» fueron variando en el tiempo, en 1933 la producción de trigo, alverjones y porotos, presenta un incremento. Debido a la abundancia de agua en estos años, los sembradores limpiaron las laderas y las planicies de «El Portezuelo», cortando los árboles y arbustos nativos que cubrían la superficie terrestre del área. En la fotografías se muestra los claros de suelos desnudos, producidos por la actividad agraria. 


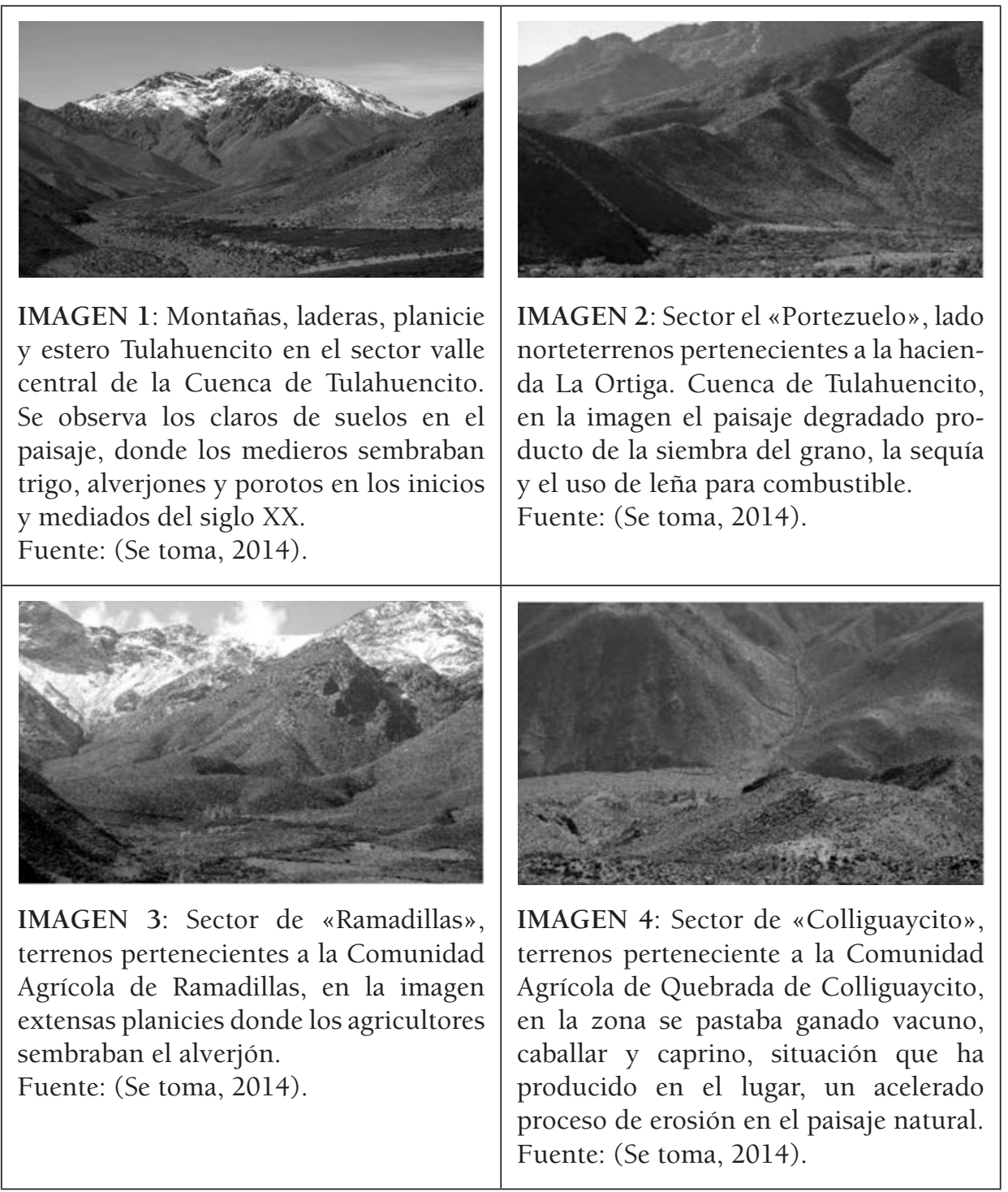

Sin embargo, durante la década de 1970, la cantidad de medieros bajó considerablemente en el sector «El Portezuelo» y la producción cayó, situación que fue provocada por el agotamiento del recurso hídrico, producido por un descenso en la precipitaciones. Según los datos pluviométricos entregados por la estación meteorológica de la localidad de Pedregal y Carén, perteneciente 
a la Dirección General de Aguas ${ }^{11}$, esta situación sumada a los problemas de administración de la hacienda La Ortiga, fueron causando la caída de la economía basada en el cultivo del grano en la Cuenca de Tulahuencito.

\subsection{La crianza del ganado vacuno y caballar en la Cuenca de Tulahuencito.}

«Casi en todas partes prevalecía la agricultura mixta, un ejemplo de esta realidad es el valle del Puangue, ubicado en el distrito de Lepe, Curacaví, María Pinto y Melipilla en la zona central de Chile, donde cerca de 700 hectáreas de tierras eran cultivadas, frente a 23.000 cabezas de ganado, de las que los vacunos representaban una mitad $»^{12}$.

La crianza del vacuno era una actividad económica que se complementaba con la siembra, el vacuno existente en la Cuenca de Tulahuencito, se utilizaba para la comercialización de carne y el aprovechamiento del cuero para la artesanía. «De los animales domésticos, el ganado vacuno es probablemente el que más contribuirá a la prosperidad de los pobladores. Este se ha visto progresar aquí considerablemente, y no podía ser de otra manera, atendiendo a los ricos pastos que abundaban en todas partes, lo mismo se puede decir del ganado caballar» ${ }^{13}$.

Los vaqueros debían bajar el ganado vacuno desde los terrenos altos de la Cuenca y de los potreros para reunirlos en los corrales, lugar donde ocurría la matanza, para la elaboración del charqui, sebo, aprovechamiento del cuero y su posterior comercialización. Los vacunos eran trasladados a la parte alta, para pastar en el paisaje de praderas, lugar donde había abundante pastos, como pucanas, coirón, pajonal, incienso y palo blanco muy nutritivas para

11 Dirección General de Aguas (2010). Datos de precipitaciones mensuales (mm), Estación Carén. Departamento de Hidrología, Subdepartamento de Meteorología y Nieve, Región de Coquimbo. Chile, Unidad de Asuntos públicos Dirección General de Aguas.

12 Góngora. M. (1956). "Casi en todas partes prevalecía la agricultura mixta, un ejemplo de esta realidad es el valle del Puangue, ubicado en el distrito de Lepe, Curacaví, María Pinto y Melipilla en la zona central de Chile, donde cerca de 700 hectáreas de tierras eran cultivadas, frente a 23.000 cabezas de ganado, de las que los vacunos representaban una mitad". Evolución de la propiedad rural del Valle del Puangue. Santiago: Universidad de Chile, Instituto de Sociología.

13 Gay, C. (1800-1873). Historia física y política de Chile, según documentos adquiridos en esta Republica durante doce años de residencia en ella y publicada bajo los auspicios del supremo gobierno por Claudio Gay, ciudadano chileno, individuo del Instituto Imperial de Francia (Academia de Ciencias de la Universidad de Chile) y de varias sociedades científicas nacionales como extranjeras, caballero de la legión de honor, Historia, Tomo Séptimo, Paris. En casa del autor Chile en el museo de Historia Natural de Santiago. 
la alimentación de las vacas, el pastoreo de los vacunos, fue produciendo un agotamiento y disminución del recurso forrajero, dando inicio a una degradación esta situación, sumada a la presión que tiene el peso de vacuno sobre el suelo, fue acelerando un proceso de erosión en las laderas y faldeos de las praderas naturales en la unidad territorial.

En la Cuenca de Tulahuencito debido a lo escabroso del relieve cordillerano, se construían grandes corrales con cercas hechas de piedra denominadas «pircas» para poder encerrar los animales, esta actividad también, transformó el paisaje en Tulahuencito. A continuación se muestra en (tabla 3), algunas cantidades de ganado y caballares.

TABLA 3

DE VACUNOS DE CABEZAS DE GANADO Y CABALLARES EN LA HACIENDA LA ORTIGA CUENCA DE TULAHUENCITO 1933, 1960 Y 1966

\begin{tabular}{|c|l|c|}
\hline Año & \multicolumn{1}{|c|}{ Ganado } & Números de vacas \\
\hline 1933 & Vacuno & 300 \\
\hline 1933 & Caballares (yeguas, mulas y burros) & 200 \\
\hline 1960 & Vacuno & 200 \\
\hline 1960 & Caballares (yeguas, mulas y burros) & 200 \\
\hline 1966 & Vacuno & 60 \\
\hline 1966 & Caballares (yeguas, mulas y burros) & 60 \\
\hline
\end{tabular}

Fuente: Jaime P. (2014).

En relación a la producción de ganado caballar, se encontraban las mulas, animales de tiro utilizados preferentemente para el acarreo de cosechas hacia los centros de acopio. Debido a la geografía escabrosa y difícil de explorar de Tulahuencito, era necesario contar con estos animales para poder realizar este trabajo, la mula es un animal que se adapta a las inhóspitas condiciones del norte semiárido, donde la sequedad produce la falta de forraje y agua para beber. Las cifras de ganado vacuno en 1933 dan a conocer importantes cantidades de rebaño presentes en la Cuenca de Tulahuencito, producto de disponibilidad del recurso hídrico, necesario para poder regar los pastizales.

Las partes altas de la cuenca eran utilizadas para el pastoreo de la vaca, comúnmente se hacían «tomaderos» junto a los canales para que los animales pudieran beber agua cuando bajaran de la montaña, en compañía de yeguas, mulas y burros que eran utilizados para la trilla.Hacia la década de 1960 la 
producción caballar y vacuna no presentó gran variación en sus cantidades, debido a la disminución de las siembras, que luego de cosechar, lo sembrado, quedaba todo el recurso forrajero para poder pastar las vacas.

La tabla 4 de cabezas de vacuno y caballar, registradas en el año 1966, muestra una baja considerable en la producción, una disminución en la disponibilidad de forraje fue desencadenando una caída en la ganadería, producto de la caída de las precipitaciones, durante los años 1967 a 1969, periodos donde el agua disponible en la Cuenca de Tulahuencito, no es suficiente para poder alimentar la masa ganadera esta realidad con el paso de los años se mantuvo, visualizando en los registro de precipitaciones un acelerado proceso de sequía en la zona.

TABLA 4

PRECIPITACIONES MAXIMAS ANUALES PERIODO 1967 A 1975 REGISTRADAS EN LA ESTACIÓN METEOROLOGICA DE CARÉN

\begin{tabular}{c|c}
\hline Años & Precipitaciones máximas anuales $(\mathrm{mm})$ \\
\hline 1967 & $33,00 \mathrm{~mm}$ \\
\hline 1968 & $27,00 \mathrm{~mm}$ \\
\hline 1969 & $13,00 \mathrm{~mm}$ \\
\hline 1970 & $30,00 \mathrm{~mm}$ \\
\hline 1971 & $29,00 \mathrm{~mm}$ \\
\hline 1972 & $100.00 \mathrm{~mm}$ \\
\hline 1973 & $50,00 \mathrm{~mm}$ \\
\hline 1974 & $15.00 \mathrm{~mm}$ \\
\hline 1975 & $47,50 \mathrm{~mm}$ \\
\hline
\end{tabular}

Fuente: DGA, (2016).

Los cambios del uso del suelo fueron transformando el paisaje natural en la Cuenca de Tulahuencito a partir de la década de los sesenta la hacienda comienza a experimental problemas económicos. El aporte de la reforma agraria tuvo por objetivo propender a un mejor uso de la tierra, permitiendo un mayor aprovechamiento de los recursos naturales. En Tulahuencito el sistema de mediero había logrado una mejor estabilidad económica en la cuenca, pues trabajaba un gran número de familias campesinas. Con la implementación de esta reforma por parte del Estado, la realidad económica de las haciendas de 
la comuna de Monte Patria empezó a cambiar, esta situación fue produciendo escasez de trabajo para las familias que vivían gracias a la siembra y la crianza del ganado vacuno.

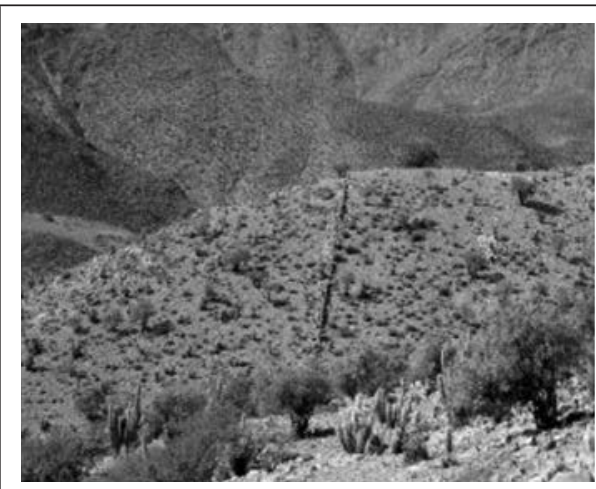

IMAGEN 5: Fotografía «Loma de la Quemada». Cuenca de Tulahuencito, una «Pirca» que cruza la loma, sirviendo de corral para agrupar el ganado caprino para su posterior matanza.

Fuente: (Se toma, 2014).

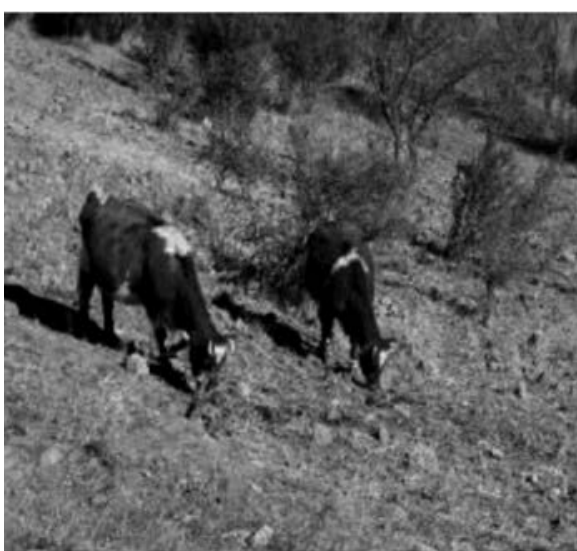

IMAGEN 7: En la imagen, el ganado vacuno presente en la Cuenca de Tulahuencito.

Fuente:(Se toma, 2014).

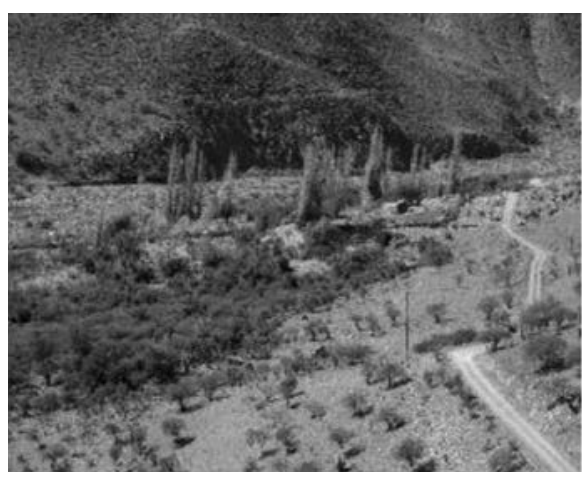

IMAGEN 6: Fotografía «Potreros del sector La Quemada». Cuenca de Tulahuencito, terrenos donde se pastaba el ganado.

Fuente: (Se toma, 2014).

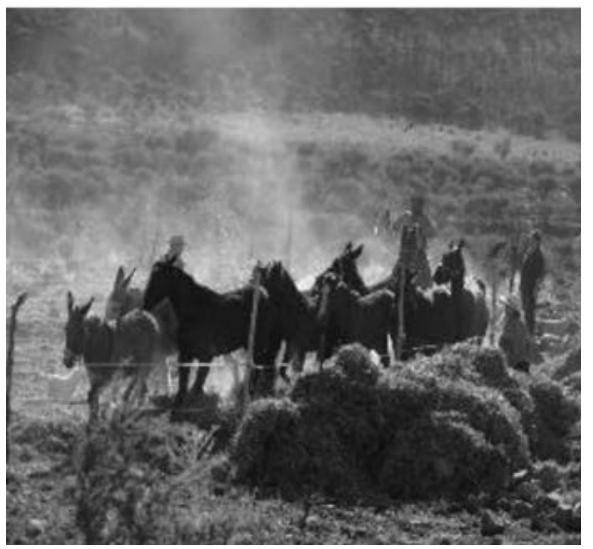

IMAGEN 8: Fotografía de uso de mulares y burros en la trilla de porotos en la Cuenca de Tulahuencito.

Fuente: (Se toma: 2014). 
En la década de los sesenta, la hacienda La Ortiga, se subdivido en varios administradores, esta provocó un desequilibrio en la producción, pues no todos los sectores eran aptos para la agricultura. Los potreros de la «Mostacilla baja ${ }^{14} » y$ «El portezuelo» empezaron a sufrir la escasez de agua estas tierras fueron abandonadas por los medieros y se convirtieron espinales y zonas erosionadas, estos lugares de la hacienda quedaron en desventaja con otras, como «El chacay» ${ }^{15}$, terrenos donde aún había agua para seguir produciendo y mantener una parte del ganado vacuno. Esta situación provocó los primeros problemas de sustentabilidad de la hacienda que se expresó en una caída en la economía de la explotación de siembra y la ganadería junto con ello un desempleo de las familias, produciendo la migración de peones e inquilinos a otros lugares del país en búsqueda de mayores oportunidades de trabajo.

\subsection{La crianza del ganado caprino y la explotación del matorral arbustivo en la Cuenca de Tulahuencito.}

«A partir del cambio modelo económico en Chile, desde 1975 en adelante, el Estado comienza a impulsar reformas económicas centradas en la liberalización de las importaciones, liberalización financiera interna, apertura de la cuenta de capital y privatización, que fueron los elementos principales hacia una economía más abierta ${ }^{16}$. Los cambios en la economía nacional fueron paulatinamente llegando a la Cuenca de Tulahuencito, los administradores de la hacienda La Ortiga, dividida en varias parcelas, decidieron ir en búsqueda de una actividad que reemplazara la siembra y el crianza de ganado vacuno y caballar, esta actividad fue la ligada a los ganados caprinos, que antes habían estado relegada por el perjuicio que producían a los cultivos. Los ganados caprinos llegan a los terrenos que antiguamente eran sembrados como «El Chacay», «El Redondo»: su nombre se origina por las características geográficas del relieve de la zona, «El Espinal»; debido a la formaciones de espinos «El Gredal»; por los gredoso de los suelo en la

${ }^{14}$ INTA, 2016. Instituto Nacional de Tecnología Agropecuaria. Consulta Atlas de Maleza. Nombre de la planta Mostacilla. Hierbas anuales o bienales, con tallos ramificados desde la base. Los lugareños de Tulahuencito, nombran la mostacilla baja aun sector de la cuenca debido la existencia de esta maleza en la parte baja del sector estudiado. En URL: http://rian.inta.gov.ar/ atlasmalezas/atlasmalezasportal/DetalleMaleza. aspx? pagante=CXC\&idmaleza=21607

15 Riedemann. P y Aldunate.G. (2014). Flora Nativa de valor ornamental, identificación y propagación, Chile zona centro.

${ }_{16}$ Ffrench, R. Satallings, B. (2001). Reformas, crecimiento y políticas sociales desde 1973. Santiago: Lom. 
superficie, «El Portezuelo» y los potreros del «Algarrobo del Tiuque». La cabra es considerada por los administradores de la hacienda La Ortiga un animal de fácil crianza, que se adapta a la sequedad y a las difíciles condiciones de subsistencia.

«La actividad ganadera presenta una larga tradición en la Región de Coquimbo, en particular, por la marcada producción de caprinos» ${ }^{17}$, esta tradición presenta su mayor esplendor con la caída de la siembra del grano y la crianza de ganado en la cuenca, provocando que los terrenos de la hacienda La Ortiga fueran arrendados para pastoreo del ganado caprino, apareciendo la figura del «criancero», que es la denominación que recibe la persona que se dedica la crianza de cabras.

Espacialmente, el ganado caprino se distribuyó por toda la unidad geográfica, formando senderos o rutas, donde el pastor con su ganado pasaban diariamente en busca de buenos pastizales, para alimentar al rebaño; la cabra busca el brote verde del rumpiato, el colliguay y el taco de los algarrobos, recurso natural disponible gracias al aporte de agua aunque escasa las lluvias y los ciclos naturales de las plantas nativas existentes en las zonas áridas. «La raza caprina predomínate en la cuenca es la criolla: esta resulta de sucesivos cruces de animales de distintas razas, adaptadas en las condiciones del medio» ${ }^{18}$. Las cabras criollas fueron las sobrevivientes de las difíciles condiciones de vivir en ambientes áridos.

La comercialización del queso de cabra, el charqui, y su carne fresca fue la fuente principal de sustento para las familias que quedaron en la cuenca, todos los años los crianceros suben a la cordillera en busca de nuevos pastos, en las denominadas veranadas en los meses octubre, noviembre, diciembre, enero, febrero y marzo, volviendo en los meses de invierno en la tabla 5 se muestra el aumento de la masa ganadera en la Cuenca de Tulahuencito.

Según el testimonio entregado por el presidente de la Comunidad Agrícola Quebrada de Minilla, Juan Luis Olivares, La Tranquita, Ramadillas en los años anteriores a 1980 la existencia de ganados caprinos en la Cuenca de Tulahuencito, era escasa en números, ya que los administradores de la hacienda La Ortiga, potenciaban la siembra y la crianza de ganado vacuno. Sin embargo a partir de la década de los ochenta la actividad caprina comienza a experimentar un cambio en forma progresiva por ejemplo en los potreros de la hacienda La Ortiga y las comunidades agrícolas arrendadas para pastar al ganado, se

17 Sánchez. A. y Morales. R. (1990). Las regiones de Chile, espacio físico y urbano-económico. Santiago: Editorial Universitaria.

18 INE (2010). Encuesta de la ganadería caprina, Las estadísticas de Chile. Santiago: ODEPA, Ediciones Instituto Nacional de Estadísticas (INE). 
forman las «majadas»: forma de asentamiento humano donde vive el criancero junto a su familia al lado del corral donde agrupa las cabras para alojar durante la noche. Junto al corral nacen los «chiqueros»: pequeños corrales construidos con ramas de romero para separar los cabritos de las cabras y así regular el consumo de leche en los animales con la finalidad de usarla para hacer queso.

TABLA 5

DE CABEZAS DE GANADO CAPRINO EXISTENTE EN LA CUENCA DE TULAHUENCITO EN LOS AÑOS 1980, 1990 Y 2010

\begin{tabular}{c|c|c|c|c|c}
\hline $\begin{array}{c}\text { Numeración } \\
\text { para cada } \\
\text { criancero al } \\
\text { año } 1980\end{array}$ & $\begin{array}{c}\text { Cabezas } \\
\text { de ganado } \\
\text { caprino }\end{array}$ & $\begin{array}{c}\text { Numeración } \\
\text { para cada } \\
\text { criancero al } \\
\text { año } 1990\end{array}$ & $\begin{array}{c}\text { Cabezas } \\
\text { de ganado } \\
\text { caprino }\end{array}$ & $\begin{array}{c}\text { Numeración } \\
\text { para cada } \\
\text { criancero al } \\
\text { año 2010 }\end{array}$ & $\begin{array}{c}\text { Cabezas } \\
\text { de ganado } \\
\text { caprino }\end{array}$ \\
\hline 1 & 500 & 1 & 600 & 1 & 240 \\
\hline 2 & 200 & 2 & 530 & 2 & 200 \\
\hline 3 & 460 & 3 & 200 & 3 & 220 \\
\hline 4 & 400 & 4 & 400 & 4 & 215 \\
\hline 5 & 380 & 5 & 300 & 5 & 180 \\
\hline 6 & 100 & 6 & 430 & 6 & 100 \\
\hline 7 & 300 & 7 & 200 & 7 & 120 \\
\hline 8 & 200 & 8 & 380 & 8 & 110 \\
\hline 9 & 340 & 9 & 330 & 9 & 130 \\
\hline 10 & 150 & 10 & 390 & 10 & 100 \\
\hline & & 11 & 210 & 11 & 110 \\
\hline & & 12 & 260 & 12 & 150 \\
\hline
\end{tabular}

Fuente: Jaime, P. (2014).

Los datos registrados en la tabla 6 , se muestra la numeración para cada criancero al año 1990, la cantidad de cabras continua aumentado, para el presidente de las comunidades agrícolas Quebrada Las Minillas, La Tranquita y Las Ramadas, Juan Luis Olivares, se mantuvieron las cantidades de cabras pero se sumó un problema: la disminución de precipitaciones.

La cantidad de milímetros registrados en la estación meteorología de Carén, pueblo cercano a Tulahuencito, da a conocer una baja en las precipitaciones máximas anuales durante los años 1990 al 1997. Esta situación genero consecuencias como falta de pastizales para mantener la producción de leche utilizada en el queso, 
principal producto comercializado. A pesar que el año 1998 se produce un aumento en las lluvias, considerando este valor como muy positivo y bueno para esta zona árida, después de este año, la escasez de lluvia vuelve el lugar.

TABLA 6

PRECIPITACIONES MAXIMAS ANUALES PERIODO 1990 A 2000. REGISTRADAS EN LA ESTACIÓN METEOROLOGICA DE CARÉN

\begin{tabular}{c|c}
\hline Años & $\begin{array}{c}\text { Precipitaciones máximas anuales } \\
(\mathbf{m m})\end{array}$ \\
\hline 1990 & $25,00 \mathrm{~mm}$ \\
\hline 1992 & $54,00 \mathrm{~mm}$ \\
\hline 1993 & $50,00 \mathrm{~mm}$ \\
\hline 1994 & $45,00 \mathrm{~mm}$ \\
\hline 1995 & $38,00 \mathrm{~mm}$ \\
\hline 1996 & $10.00 \mathrm{~mm}$ \\
\hline 1997 & $33,00 \mathrm{~mm}$ \\
\hline 1998 & $89.00 \mathrm{~mm}$ \\
\hline 1999 & $19,50 \mathrm{~mm}$ \\
\hline 2000 & $66.50 \mathrm{~mm}$ \\
\hline
\end{tabular}

Fuente: DGA, (2016).

Ante esta situación, el criancero se vio obligado a elaborar una estrategia para contar con forraje disponible para su ganado: los cabreros empezaron a arrendar los potreros de la hacienda La Ortiga junto con las acciones de aguas de los canales para regar, actividad que fue generando en los terrenos de la hacienda el surgimiento de pastizales, donde crecía el pasto cola de ratón, trébol, alfalfa, alfilerillo, el romero, mollaca y las formaciones de espinales.

Por su parte, para el presidente de la comunidad Agrícola Quebrada Colliguaycito, Pedro Jaime, la explotación del ganado caprino se convirtió en el sustento económico para las familias campesinas de Tulahuencito, apellidos como los Valderrama, Muñoz, Castillo, Cortés, Toledo, Michea, Olivares, Palleros, Tapia, Mujica y los Villalobos se convirtieron en los crianceros dueños de los ganados que poblaron la cuenca. Hacia el año 2010 la crianza de ganado siguió su desarrollo a pesar de una leve disminución de las cabezas de ganado caprino, producto de la ausencia de forraje para las cabras, los potreros arrendados a la hacienda La Ortiga, para el regadío se fueron poblando de 
espinales y el suelo debido al paso de agua fue perdiendo materia orgánica, provocando el empobrecimiento y la erosión impidiendo el crecimiento de abundantes pastizales para pastar el ganado y así mantener la producción de queso de cabra, ante esta situación el paisaje de Tulahuencito, a causa del ganado caprino, ha sido alterado, por el sobrepastoreo caprino.

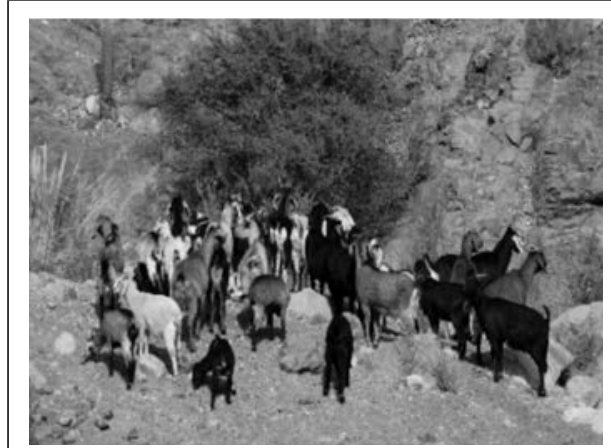

IMAGEN 9: En la imagen, un grupo de cabras, en el sector de ríicito presente Cuenca de Tulahuencito, comuna de Monte Patria, Región de Coquimbo. Fuente:(Se toma, 2014).

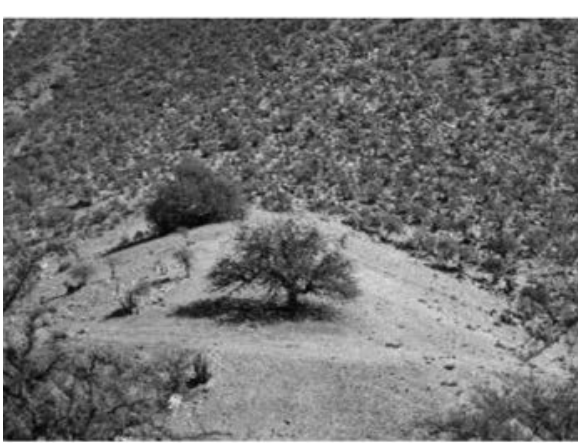

IMAGEN 10: «Loma de Colliguaycito» terrenos de la Comunidad Agrícola Quebrada de Colliguaycito, Cuenca de Tulahuencito, comuna de Monte Patria, Región de Coquimbo.

Fuente:(Se toma, 2014).

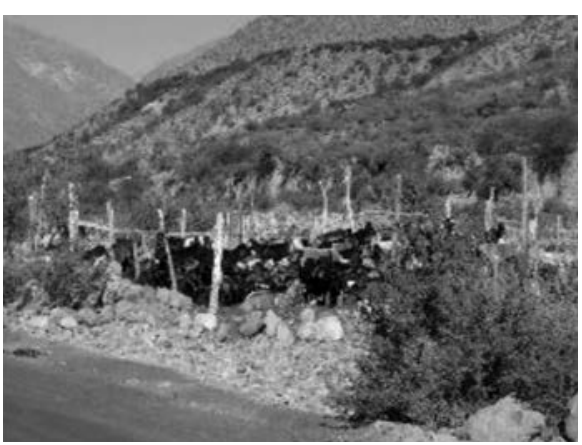

IMAGEN 12: Corral de ganado caprino, ubicado en el sector Pedregal Oriente, inserto en la Cuenca de Tulahuencito, comuna de Monte Patria, Región de Coquimbo.

Fuente:(Se toma, 2014). 
Otra de las actividades que surge con el cambio de modelo económico y la caída de la agricultura y la crianza de ganado vacuno, es la explotación de matorral arbustivo de la Cuenca de Tulahuencito. Las personas comenzaron a emplear la vegetación nativas, para ser utilizada como leña para la cocina y carbón para comercializar.

\section{CONCLUSIONES}

En paisaje natural de la cuenca, ha sido transformado por las actividades económicas que se dieron en la zona rural. La hacienda dio vida a una sociedad rural marcada por la explotación de la tierra, la figura del campesino y el mediero fueron dando vida y transformado el entorno natural del área de estudio. El desarrollo rural y la trasformación del paisaje en la Cuenca de Tulahuencito fue acompañada por los procesos culturales y por diversos modelos económicos.

El paso de los años vino acompañado de la llegada de procesos históricos, como la Reforma Agraria y la subdivisión de la hacienda La Ortiga, acontecimientos que influyeron en la economía del latifundio, situación que provocó el desempleo y la migración de familias a otros lugares en busca de mejores oportunidades. Esta situación sumada a la conformación de las comunidades y la incorporación de un nuevo modelo económico de libre mercado, trajeron consigo la crianza del ganado caprino, actividad que ha proliferado y que permite a las comunidades del lugar generar los ingresos económicos para sustentar los hogares.

En la actualidad la Cuenca de Tulahuencito sigue siendo un lugar donde el paisaje rural requiere de una planificación que promueva un desarrollo en forma sostenida que permita a los pequeños crianceros y agricultores quedarse en el lugar y evitar que las propiedades que ellos arriendan sean vendidas a la gran industria del monocultivo.

\section{BiBLIOGRAFIA}

Aylwin, M., Bascuñán, C., Correa, S., Gazmuri. C., Serrano, S. y Tagle. M. (1994). Chile en el siglo XX. Santiago: Quinta edición Editorial Planeta.

Arancio, G., Muñoz, M y Squeo, F. (2001). Descripción de algunas especies con problemas de conservación en la IV Región de Coquimbo, Chile. En Libro Rojo de la Flora Nativa y los sitios Prioritarios para su Conservación. La Serena: Ediciones Universidad de La Serena. 
Bengoa, J. (1990). Haciendas y campesinos, Historia social de la agricultura chilena. Tomo II. Santiago: Ediciones SUR.

Biblioteca del Congreso Nacional de Chile (2017). Glosario de términos geográficos. Significado de "Portezuelo": Sección baja o deprimida situada entre dos relieves mayores. En URL. https://www.bcn.cl/siit/glosario/index_html visto 09 de enero 2017.

Carmagnani. M. (1998). Desarrollo industrial y subdesarrollo económico el caso chileno (1860-1920). Santiago, Chile: Dirección de bibliotecas, Archivos y Museos. Departamento de Ciencias Históricas Universidad de Chile y Centro de Investigaciones Diego Barros Aranas.

CONAMA, (2006). Ficha de estado de conservación de Prosopis chilensis URL: http:// www.mma.gob.cl/clasificacionespecies/fichas9proceso/FICHAS_INICIO_9o_PROCESO_PDF/Prosopis_chilensis_2711.pdf

Ffrench, R. Satallings, B. (2001). Reformas, crecimiento y políticas sociales desde 1973. Santiago: Lom.

Gay, C. (1800-1873).Historia física y política de Chile, según documentos adquiridos en esta Republica durante doce años de residencia en ella y publicada bajo los auspicios del supremo gobierno por Claudio Gay, ciudadano chileno, individuo del Instituto Imperial de Francia (Academia de Ciencias de la Universidad de Chile) y de varias sociedades científicas nacionales como extranjeras, caballero de la legión de honor, Historia, Tomo Séptimo, Paris. En casa del autor Chile en el museo de Historia Natural de Santiago. Góngora. M. (1956). Evolución de la propiedad rural del Valle del Puangue. Santiago: Universidad de Chile, Instituto de Sociología.

Hoffmann, A. (2012). Flora silvestre de Chile, zona central. Quinta edición. Santiago: Ediciones FUNDACIÓN CLAUDIO GAY.

INE (2010). Encuesta de la ganadería caprina, Las estadísticas de Chile. Santiago: ODEPA, Ediciones Instituto Nacional de Estadísticas (INE).

INE (2012). Reportes Estadísticos comunales, población de la comuna de Monte Patria, Región de Coquimbo, Chile. Santiago: Biblioteca del Congreso Nacional de Chile, Comuna de Monte Patria, Provincia del Limarí, Región de Coquimbo.

INTA, 2016. Instituto Nacional de Tecnología Agropecuaria. Consulta Atlas de Maleza. Argentina.URL:http://rian.inta.gov.ar/atlasmalezas/atlasmalezasportal/DetalleMaleza.aspx? pagante $=C X C \& i d m a l e z a=21607$ Visto 10 de enero2017.

Plan regional de gobierno 2014-2018. (2014). Región de Coquimbo. División de Planificación y Desarrollo Regional. Gobierno Regional de Coquimbo

PNUD (2011). Uso eficiente de leña y otras energías alternativas en comunidades rurales. Santiago: Programa de Recuperación Ambiental Comunitario.

Ridermann, P. y Aldunate, G. (2003). Flora nativa de valor ornamental, identificación y propagación, Chile zona central. Santiago: Editorial Andrés Bello.

Sánchez A. (2005). Geografía de Chile. Santiago de Chile. Biblioteca Internacional.

Sánchez. A. y Morales. R. (1990). Las regiones de Chile, espacio físico y urbano-económico. Santiago: Editorial Universitaria. 
Toso, R. y Feller, A. (1983). Serie de estudios económicos, Documentos de Investigación. $N^{\circ} 21$ La crisis económica de la década del treinta en Chile lecciones de una experiencia. Santiago: Departamento de informaciones, estadísticas y publicaciones del Banco Central de Chile.

Toledo, X. y Zapater, E. (1991). Geografía General y Regional de Chile. Santiago: Editorial Universitaria.

Dirección General de Aguas (2010). Datos de precipitaciones mensuales (mm), Estación Carén. Departamento de Hidrología, Subdepartamento de Meteorología y Nieve, Región de Coquimbo. Chile, Unidad de Asuntos públicos Dirección General de Aguas.

\section{TESTIMONIOS ORALES}

Jaime P. (2014). Testimonios orales del presidente de la Comunidad Agrícola Quebrada Colliguaycito, comuna de Monte Patria, provincia del Limarí, Región de Coquimbo.

Olivares J. (2014). Testimonios orales del presidente de la Comunidad Agrícola Quebrada de Minilla, La Tranquita y Ramadillas, comuna de Monte Patria, provincia del Limarí, Región de Coquimbo.

Fecha de recepción: 2 de diciembre de 2015.

Fecha de aceptación: 30 de octubre de 2017. 